\title{
The relatives' voice: how do relatives experience participation in reablement? A qualitative study
}

This article was published in the following Dove Press journal:

Journal of Multidisciplinary Healthcare

28 December 2016

Number of times this article has been viewed

\author{
Kari Margrete Hjelle ${ }^{1,2}$ \\ Herdis Alvsvåg ${ }^{3}$ \\ Oddvar Førland ${ }^{2,3}$ \\ 'Department of Occupational \\ Therapy, Physiotherapy, and \\ Radiography, Faculty of Health and \\ Social Sciences, ${ }^{2}$ Centre for Care \\ Research Western Norway, Bergen \\ University College, ${ }^{3}$ Faculty of Health \\ Sciences, VID Specialized University, \\ Bergen, Norway
}

Background: Reablement is an early and time-limited home-based model of rehabilitation intervention with an emphasis on intensive, goal-oriented, and multidisciplinary assistance for persons experiencing functional decline. When rehabilitation in general takes place in the person's own home, in contrast to an institution, relatives may have larger responsibilities in helping and supporting the family member. Although there is evidence, showing that family caregivers, such as spouses and children, experience burdens and demanding situations related to their caregiving role, there are currently few publications exploring relatives' experiences of participating in reablement. The aim of our study was to explore and describe how relatives in a community setting in Norway experienced participation in the reablement process.

Methods: Six relatives participated in semi-structured interviews. Qualitative systematic text condensation was used as the analysis strategy.

Results: Five themes emerged that summarized the relatives' experiences with reablement: 1) a wish to give and receive information, wish to be involved; 2) wish to be a resource in reablement process; 3) conflicting expectations; 4) have more free time to themselves; and 5) a lack of follow-up programs. Conclusion: Our findings highlight the involvement and collaborative process between health professionals, older adults, and relatives and have practical significance for health care services. To advance collaborative practices, the municipal health and social care services should consider establishing a system or a routine to foster this collaboration in reablement. Follow-up programs should be included.

Keywords: family caregivers, involvement, system of collaboration, follow-up programs, conflicting expectations

\section{Background}

Reablement is an early and time-limited home-based intervention with an emphasis on intensive, goal-oriented, and interdisciplinary support and assistance for older adults experiencing functional decline. ${ }^{1}$ There has been growing international interest in reablement or restorative care as a new rehabilitative approach for the elderly. ${ }^{1,2}$ When rehabilitation takes place in the home, as opposed to an institution, relatives often become more involved and have an important role as physical and emotional supporters in promoting the rehabilitation process. ${ }^{3}$

The development of reablement in Norway started in 2012, and to our knowledge, to date $\sim 40 \%$ of the municipalities have implemented it as one specific model of organizing rehabilitation services. In Norway, rehabilitation is defined as timelimited, planned processes with clear goals and measures, where several providers collaborate in assisting the person's own efforts in achieving the optimal level of
Correspondence: Kari Margrete Hjelle Department of Occupational Therapy, Physiotherapy, and Radiography, Faculty of Health and Social Sciences, Bergen University College, Post Box 7030, Bergen 5020, Norway

Tel +475 977| |34 I

Email Kari.Hjelle@hib.no 
coping of everyday activities, functional ability, independence, and social participation. ${ }^{4}$ Reablement complies with all the criteria described in this definition. However, reablement means home-based rehabilitation and is not institution- or hospital-based. A coordinated multidisciplinary team and home-care services work together with the older adults toward shared goals, which is one of the main characteristics of reablement. ${ }^{1,2,5}$

International and national political documents on health care and rehabilitation increasingly focus on the need and expectation to include relatives in the rehabilitation process in the years to come. ${ }^{3,6-9}$ Consequently, these documents directly indicate that relatives have a central role in the formulation and implementation of their family members' health care. ${ }^{3,6,7}$ In this type of cooperation, the knowledge and resources of the family member, the relatives, and the professionals are all valuable integral components of the rehabilitation process. Furthermore, it is emphasized that the community should have dialogue with all parties involved in this process. As stated in the WHO's Framework for Action, ${ }^{7}$ collaborative practices will become more embedded, thereby strengthening health care systems and improving health outcomes. The framework ${ }^{7}$ emphasizes that collaborate practice occurs when health care professionals in communities collaborate with patients and their families to deliver the highest quality of care. With this collaborative practice, the person or the patient, the relatives, the professionals, and the politicians are all important persons who can play a role in changing and advancing collaborative practices in the years to come. ${ }^{7,8}$

Although the importance of involving informal caregivers is highlighted in official documents, research points out that health care professionals contact and collaboration with informal caregivers is often lacking. ${ }^{10-14}$ In the study of van Wieringen et al, ${ }^{10}$ "professionals generally regard caregivers as 'coworkers' because both parties perform comparable tasks". However, informal caregivers do not feel like coworkers because they are hardly involved in the decision-making about care. Moreover, van Wieringen et $\mathrm{al}^{10}$ argue that caregivers can experience a lack of sympathy and support from professionals. This usually results from the limited knowledge that professionals have of caregivers' needs and attitudes toward care. Overall, it can be said that professionals do not always interact with caregivers. ${ }^{10}$

Nolan ${ }^{15}$ summarized the available studies on the cooperation between relatives and rehabilitation teams, with a particular focus on families and relatives who are new to the role. He notes that many individuals take on this role without having a real choice and without being aware of the range of their options. They did not receive the information and opportunities to acquire the needed skills, and thus they were not quite prepared for their role of the caregiver. In fact, families were rarely as fully involved in the rehabilitative process as they could be, and thus they were often marginalized. Nolan ${ }^{15}$ highlights that this situation is not unique to rehabilitation but rather reflects the general failure to engage family caregivers across health and social services.

The literature from different parts of the health care sector explores the issues of familial ties, gendered expectations of family caregivers, and the social and moral pressure to provide care. ${ }^{12,16,17}$ In the study of Cree et al, ${ }^{12}$ many carers perceived a lack of involvement in care planning and a lack of recognition and appreciation of their role from health professionals. Barriers to involvement included structural barriers, such as the timing and location of meetings, cultural barriers relating to power imbalances within the system, and specific barriers relating to confidentiality. Greenwood and Mackenzie, ${ }^{13}$ highlighted the importance for health care professionals to acknowledge the impact of caring, and of carers' reactions to it. If clinicians are aware of the carers change in roles and relationship and in their sense of identity, they will be better equipped to support carer's emotional and practical needs. Furthermore, Sadler and McKevitt ${ }^{17}$ pointed out that recent interventions have moved away from offering advice and support to caregivers or enhancing their psychological competence, aiming instead to train them in such skills as personal care, feeding, swallowing problems, transfers, and mobility activities, that caregivers are assumed to require. In addition, Larkin and Milne ${ }^{18}$ provide a critical reflection on carer empowerment in the UK, an issue which has received limited attention in policy and research. Despite increased national acknowledgment of carers, a politically active carers' movement and a number of policies intended to enhance the recognition and rights of carers, many carers remain invisible and receive little support from health care services.

There are currently few international publications exploring relatives' experiences with participating in reablement. In a study by Wilde and Glendinning, ${ }^{2}$ a number of relatives had the experience of not being included in the reablement process. Several of them reported having heavy caring responsibilities that were hard to bear, and needing support and a break from providing care. However, some informal carers reported learning structured and new ways of meeting the family members' needs. Involving and supporting relatives in reablement can enhance the relatives' well-being and can benefit the carer by being able to perform and participate in valued activities. ${ }^{2}$ Glendinning et a ${ }^{19}$ state that some relatives expressed the pressures of the emotional and practical help they provided to their wives, and were disappointed that they 
received no support or advice from the reablement team. A study by Rabiee and Glendinning ${ }^{20}$ revealed that the family members were primarily concerned with the assurance that their older parents would receive care and assistance, and for some relatives, this was more important than training their parents to live independently.

As seen, empirical studies of relatives' experiences with participating in older adults' reablement have been studied to a limited extent. There is a gap in our knowledge regarding relatives' experiences with participation in their family members' reablement processes. Thus, the aim of our study was to explore and describe how relatives in Norway perceive this participation.

\section{Methods Design}

The study was designed as a qualitative phenomenological study, ${ }^{21,22}$ which aims to gather data of events from the people concerned and in their own words. The analysis is based on decontextualization and recontextualization, which represents an inductive method of reasoning.

Ethical approval was granted by the Norwegian Social Science Data Services (NSD) (NSD 2014/37944). The transcribed data are stored on a secured Research Server at the Bergen University College. Only the first author has access to the data on this server. These data are highly personal and are not shared, according to the guidelines from Regional Committees for Medical and Health Research Ethics in Norway (REK).

\section{Participants and context}

The current study is part of a larger research program on reablement in home-dwelling adults which includes a randomized controlled trial in a rural municipality in west Norway with $\sim 14,000$ inhabitants. ${ }^{5,23,24}$ Participants were recruited according to inclusion criteria. The inclusion criteria stated that the participants were relatives (spouse, child, or other kinship), caring for older family members living at home. The family members had participated, or were participating in reablement services from the local community. The participants had contact and visited their family members on a regular basis. At last, the inclusion criteria stated that the participants had capacity to consent and were interested in sharing their experiences in relation to the topic under consideration.

The participants were recruited by the local project leader of the reablement research program. The leader asked first the family member for permission to invite the relative to take part in the study. A few family members hesitated, which was respected. The local project leader confirmed with relatives that the family member accepted, and further met the inclusion criteria to participate in the study. All the relatives asked, accepted to be part of the current study.

The sample included two men and four women aged 40-70 years (Table 1). The staff of the reablement service obtained the participants' written informed consent before the interviews started. The sampling decisions are further outlined in the methodological considerations.

\section{Reablement intervention}

The reablement intervention lasted for a maximum of 3 months for each older adult person. The persons had different diagnoses, prognoses, and functional level. The intervention was tailored to the older adults' goals, and thus the components of the rehabilitation plan varied as described in Table 2. ${ }^{23}$ As part of the baseline assessments, the Canadian Occupational Performance Measurement interview was conducted by an occupational therapist or physiotherapist to identify the older adults' valued goals for the reablement. ${ }^{24}$ The goals were related to the older adults' ability to cope with and participate in important everyday activities at home or in society. Based on these goals, the integrated team discussed how the reablement process should be tailored to support and assist the older adults in achieving their goals during the rehabilitation period. Thus, the components of the intervention varied and consisted of both general and individual features, as described in Table $2 .^{23}$

\section{Data collection method}

Baseline demographic data were collected from all participants before starting the interviews, including age, gender, relationship to family member receiving reablement service, and the geographical distance between the participant and the family member (Table 1 ).

Table I Characteristics of participants $(\mathrm{N}=6)$

\begin{tabular}{lllll}
\hline Gender & Age & Relationship & Gender & $\begin{array}{l}\text { Distance to person } \\
\text { undergoing reablement - } \\
\text { relatives (miles) }\end{array}$ \\
\hline Female & $40+$ & Daughter & Female & 66.5 \\
Female & $50+$ & Aunt & Female & 0.62 \\
Female & $60+$ & Daughter & Female & 43.8 \\
Female & $60+$ & Spouse & Male & - \\
Male & $60+$ & Son & Female & 0.62 \\
Male & $70+$ & Spouse & Female & - \\
\hline
\end{tabular}


Table 2 Features of the reablement intervention in our study ${ }^{23}$

General features
- The rehabilitation period lasted a maximum of 3 months.
- An occupational therapist or physiotherapist conducted the COPM
interview and developed the rehabilitation plan together with
the participant based on the identified activity goals. Thereafter,
an integrated multidisciplinary team with shared goals guided the
participant during the whole rehabilitation period.
- In addition to home-care personnel-assisted training, a minimum of
I hour physiotherapist and/or occupational therapist-assisted training
each week.
- The treatment involved repetitive training and multiple home-visits
by health care personnel, who were present during daily training for
the purposes of building confidence and relearning skills.
- All health care personnel stimulated the participant in self-
management and self-training.

\section{Individual features}

- Training in daily activities, such as dressing, food preparation, vacuuming, bus transport, visiting friends at a club, or being able to knit.

- Adaptations, such as advice on appropriate assistive technology or adapting the activity itself or the environment to simplify activity performance.

- Exercise programs, such as indoor or outdoor walking with or without walking aids, climbing stairs, transferring, and performing exercises to improve strength, balance or fine motor skills. The exercises were incorporated into daily routines and the person was given a manual explaining each of the exercises and encouraged to train on their own.

Note: Reproduced from @ Tuntland et al; licensee BioMed Central. 20I4. Creative Commons license and disclaimer available from: http://creativecommons.org/licenses/ by/4.0/legalcode. ${ }^{23}$

Abbreviation: COPM; Canadian Occupational Performance Measurement.

\section{Semi-structured interview}

The purpose of the interviews was to explore how relatives in Norway experience their participation in reablement. The interviews were carried out as soon as participants consented to take part in the study. The data were obtained from interviewing participants face-to-face in their own homes, or in other places well suited for interviews. The interviews were conducted by $\mathrm{KMH} \sim 1$ month after the family members' reablement process had been completed.

A semi-structured interview guide was developed according to Kvale and Brinkmann. ${ }^{25}$ To explore the relatives' experiences in detail, descriptive questions were used, ${ }^{22,26}$ such as "How did you experience your participation in reablement?" and "Would you please give some examples of how you collaborated with the reablement team?" (Box 1).

Each interview lasted from 60 to 90 minutes. The interviews were conducted between June 2014 and June 2015. The data were digitally recorded and later transcribed verbatim by the first author.

\section{Data analysis}

The data was analyzed according to and inspired by phenomenological decontextualization and recontextualization. ${ }^{27}$ Qualitative systematic text condensation was used as the analysis strategy. ${ }^{27} \mathrm{KMH}, \mathrm{HA}$, and OF separately and together analyzed the transcribed data using an inductive approach with a four-step analysis procedure. ${ }^{27}$ Step 1, total impression: from chaos to themes. Step 2, identifying and sorting meaning units: from themes to code groups. Step 3, condensation: from code to meaning. Step 4, synthesizing: from condensation to descriptions and concepts. ${ }^{27}$
Box I Topics included in the interview guide

Please, tell me how did you experience your participation in reablement? Give examples.

How did you get information and knowledge about the reablement service?

How did you collaborate with the reablement team?

How do you want to collaborate with the reablement team?

How did you experience your family member's participation in reablement?

In the first step of the analysis, the authors read the interview transcripts to obtain a general sense of the overall data. The transcriptions were read and discussed several times to gain a deeper understanding of the data. A preliminary analysis was begun to identify some topics. ${ }^{27}$

In the second step of the analysis, we identified "meaning units", which were text fragments that reflected information about the participants' experiences of reablement. Then, we began coding by identifying and sorting these meaning units. A meaning unit encompassing the experiences of participating in reablement was, for example, coded as lack of involvement. We went back and forth between the data and the codes to examine the existing codes and add new ones. During this phase of decontextualization, we reflected on the similarities and differences of each code. The final codes were based on the consensus of all authors after reading all the transcripts. ${ }^{27}$

The third step of the analysis involved the systematic abstraction of meaning units within each of the code groups established in the second step of the analysis. The transcripts were read systematically to identify and classify the meaning units into thematic code groups. The meaning units were translated into the researchers' language. ${ }^{27}$ 
In the fourth step of the analysis, the data were recontextualized by developing descriptions, providing stories that reflected the entirety of the original context. This step was outlined in themes, and representative text excerpts from the transcripts are included as quotations in the reporting of the results. ${ }^{27}$ The quotes could be better contextualized using specific data from the participants (i.e., man, woman, daughter, spouse, and age); however, we decided not to use quotations to maintain the anonymity of the few participants. This decision is further outlined in the methodological considerations.

\section{Results}

The results from the interviews identified five themes, which are each illustrated by quotations from the data. The themes represent the relatives' most important experiences with reablement: 1) a wish to give and receive information, wish to be involved; 2) wish to be a resource in reablement process; 3 ) conflicting expectations; 4) have more free time to themselves; and 5) lack of follow-up programs.

\section{Wish to give and receive information, wish to be involved}

To give and receive information was identified as a strong feature of the relatives' wish to be involved in the reablement process. The relatives stated that they had a substantial amount of knowledge about their family members that could complement the professionals' knowledge. Though some relatives were satisfied with the information and dialogue they had with the reablement team, most of them felt that they were not invited to provide information about their close family members. One participant stressed that if she had known about her family member's goal of going downstairs to the basement to wash clothes, she would have protested. She said this:

We discovered that one of the goals of our family member's reablement was to go down the basement stairs to wash clothes. As family we disagreed with this goal, because in our opinion the basement stairs are steep and dangerous for her. We wanted her to have a washing machine in the kitchen. I think it is necessary and important that the reablement service collaborate with the relatives and request their opinion of the goals. It is not always that the relatives are right, however it is important to discuss all perspectives.

The relatives would have preferred an invitation to a conversational meeting to provide information about the situation and to jointly find other possible solutions. The relatives also expressed a wish to be informed about how to support and motivate their family members to engage in physical exercise and perform everyday activities. This desire was expressed as "I am dependent on the information about what and how I can contribute." Some relatives did not receive any information from the reablement team but were informed by the family member undergoing reablement or by reading general information on the Internet. Furthermore, several relatives wanted more information about the reablement service. For example, a relative expressed:

I did not receive any information, it was my mother who got the information, and she called me and asked what to do. However, I could not give her any advice, since I did not know anything about this reablement. I wanted a phone call from the reablement team to learn more about it. In addition, I could give them some important information. Still, my mother could make decisions herself.

Although the relatives were very satisfied with their family members' reablement, some of them called for an opportunity to provide input on the content of the reablement process. To be involved in the reablement process, the relatives wanted a system, a routine, an automatic process, a culture, and an attitude of giving and receiving information that was valuable to all parties of the reablement process. Here is an example:

Even though the family members have to make their own decisions, the relatives need to have information. Of course, I could have gotten some information from the information file at home, and I could have asked more questions to the reablement team. It may be that the reablement team perceived my mother as well orientated, with no need to involve the relatives. However, I think that my mother says that she has to call her daughter before she says yes to anything. I really think it would be good for all parties that the reablement service phoned the relatives, it ought to be a regular routine, a system. In addition, it would be fine to have a meeting where all parties involved are present, also my mother. I am not sure my mother is always honest with the health care personnel, and I am not sure she tells everything.

Several of the participants confirmed that they wanted to share and discuss information and knowledge about their family members with the reablement team. One participant stated this:

I want to be in a dialogue with my mother and with the system. It has to be a system that automatically invites persons involved to meetings.

However, another participant said it was not always possible for her to travel the distance to this meeting place in her mother's community due to time-constraints and her job situation. In fact, a phone call would be preferable. She said further: 
Due to the distance between my home-place and my mother's home, I prefer a phone call, just to give and receive information. My mother is quite orientated and takes care of herself in daily life. However, if I had been invited to a first meeting, I could have information about my mother's reablement, and have the opportunity to give some information, or tell them about my view of mother's situation. It does not cost that much to have a system of information.

Another participant living in the same community as the family member taking part in the reablement service, also wanted a phone call. She highlighted that the reablement team have the responsibility to take a phone call to ask how the relatives see the family member's situation. Further, she said:

It is a strength that several people see the older person's situation from different perspectives. And the professionals need to look at families as a resource. The relatives know their family member quite well.

In summary, the relatives experienced that they were not invited by the reablement service to take part in an open dialogue to give and receive information and knowledge about their parent's or spouse's reablement process. This they perceived as a lack of system and routine to invite relatives to participate in the family member's reablement process.

\section{Wish to be a resource in the reablement process}

The relatives in our study highlighted that they wanted to be a resource in the reablement process. Several relatives said they wanted to reinforce the professionals' work by supporting and motivating the family member in the training and everyday activities. One of the relatives said this:

I think it is important for me to motivate my mother, and I think I'll do this better if I had some information about how I can support and encourage her. If the relatives were a part of the team, we could have reinforced the team's work by our encouragement in their struggle to achieve better function in daily activities.

Being a resource in the reablement team, some of the relatives wanted to have an important role and work in the same direction as the reablement team. Though this collaboration requires more time and resources from all parties involved, the relatives felt this was necessary to have a successful rehabilitation. The collaboration depends on respect for each other's knowledge and role in the reablement process. This notion was expressed as:
It is necessary to give the relatives an important role in the team and give them encouragement to share their opinions and knowledge. The relatives have a lot of knowledge of the family member, as they have close relationship with them. To know a person is essential knowledge and provides a great resource for the team. The professionals have to meet the relatives in a respectful way and give them the feeling of being important and that they contribute to the team.

To initiate the collaboration, several of the relatives said a telephone call from the reablement team is necessary. The focus of this early conversation could be, for example, the relatives' opinions of the family members' situation. One participant said:

It is very good to have more people looking at the situation; we see different things, and that's great. It is really a strength.

\section{Conflicting expectations}

The relatives wanted to be involved in the family member's reablement process and collaborate with the reablement team. However, conflicting expectations appeared as an essential feature of involvement. These conflicting expectations were about: expectations of oneself, of the reablement service, of the family members participating in the reablement process, and of the reablement content. Conflicting expectations created boundaries for relatives' involvement in the reablement process. One participant stressed this in the following:

My mother cannot expect me to come here more often; I cannot expect it of myself, and the health service cannot expect it of me.

To clarify the expectations from different parties, it was important for some relatives to make a conscious choice in terms of how to be involved in the reablement process. This quotation is an example:

I intentionally did not stay with my mother in the beginning of her reablement. I only stayed the two first days before the reablement team started. She could not be dependent on me staying in her house. I don't want it, I don't have the capacity, and I don't think it's right. Being alone as a relative, I had to set limits.

Although the expectations were clarified of how to be involved in reablement, all the expectations could be a burden, as quoted in the following:

However, it is all the burdens; I should have done that, and I should have done this. Knowing my mother had contact with the reablement team was a great relief. 
Likewise, another participant also expressed feeling burdened by having several people in the family who needed help and support, despite their age. The totality of helping others might be perceived as a burden. Although all of the relatives in our study wanted to collaborate with the reablement team and take part in the meetings, the expectation of being present at all meetings might also be a burden. However, one solution to the relatives' conflicting expectations was having the opportunity to make decisions without feeling badly. One participant claimed:

I want to be invited to take part in the meetings, to share my knowledge and ask questions. However, I want to decide myself if I have the opportunity to take part, and I don't want to have a bad conscience.

The relatives wanted to be involved; however, some of them wanted to have the freedom to choose how much and when they could be involved.

Though the relatives preferred being invited to collaborate with the professional team and having an important role, their conflicting expectations might be an important burden to navigate.

\section{Free time}

The relatives experienced the reablement team's presence in the home as a significant relief, and free time for other tasks. According to one participant, the reablement team cared for his mother and motivated her to perform everyday activities and physical exercises. He said:

The reablement team in a way shared the responsibility of caring for my mother with me and that gave me some free time. That was really an unexpected relief, in a way an unexpected effect of reablement.

The free time was a break from providing care and an opportunity for the relatives to relax. They knew that health care personnel were in the house and had the responsibility for supervising and supporting the family members performing everyday activities and doing physical training. The relatives had the opportunity to leave the house without worrying about the person being alone. Knowing that health professionals were in the house, the relatives experienced this as an opportunity to be reenergized. One participant said:

I got some time off for myself; that is scarce, I got some new energy.

\section{Lack of follow-up programs}

Several of the participants noted the lack of follow-up programs for the family member as a strong missing feature of reablement. The relatives described how they were very concerned about the family members' health condition when reablement ended. They missed a routine or a system of follow-ups to ensure that the persons' achieved function were maintained. The reablement lasted up to 3 months, and then it suddenly ended. Below is a description of a relative's experience of the abrupt ending of the family member's reablement:

I would really prefer a smoother transition after the three months of reablement. The system should not leave them so suddenly. I think that the sudden ending is no good, because there is a loss when the professional is no longer motivating the family member to train and practice the obtained skills. The professionals do not have to come every day; however, one or two days providing motivation for further training and practicing everyday activities is fine. I really want to say that we have been very satisfied with this reablement program, but the ending was too abrupt. Suddenly, there was nothing. It is not that easy training alone all the time.

Some of the relatives had suggestions for an individualized follow-up program. This program could include a phone call or a short home visit from the reablement team to ask how their training is proceeding. Several of the relatives thought that checking about the family members' health situation would motivate them to continue training. One participant said:

Many older adults don't have someone who asks if their training is continuing and in that way, it is a motivator.

Though the lack of a follow-up program was identified as a strong characteristic of several of the relatives' experiences with reablement, some older adults did not want a follow-up program. One participant said that their family member had the opportunity to attend a day center. However, though the family member lived alone, she did not want to accept this offer. She had her social network and wanted to go to the café as usual with her friends, meeting people on the way. In this manner, she had intrinsic motivation to train herself by performing everyday activities and participating in society as she did before reablement.

Summing up, the findings of this study showed that the relatives perceived there was a lack of system and culture of being involved, being seen as a resource and collaborating with the reablement team during the reablement process. However, it was important to clarify conflicting expectations from different parties and make a choice of how to be involved. When health care professionals from the reablement team were in the house, the participants experienced 
this as a free time, and a break from providing care. Several of the relatives experienced this as an unexpected relief, a safety net, and an opportunity to gain energy. The transition from reablement was too abrupt and the relatives felt that an individualized follow-up program was missing.

\section{Discussion}

The aim of this study was to explore and describe how relatives experience their participation in reablement. The relatives in our study expected more information from the reablement team than what was given. These findings are confirmed by several researchers, ${ }^{10,12,16,28,29}$ who argue that how the role as the relative in health care is handled is strongly associated with the opportunity to obtain information. Studies by Glendinning et $\mathrm{al}^{19}$ and Wilde and Glendinning ${ }^{2}$ revealed that family caregivers felt more confident in their caring responsibilities when information about the management of daily routines was passed on from the reablement team and when advice related to their own needs was given.

\section{Wish to give and receive information; wish to be involved}

One of the main results in our study was that the relatives were not invited by the reablement service to collaborate with the team to work together to enhance the older adult's reablement process. For example, some relatives wanted to be involved in the family members' goal-setting. The older adult's own understanding of which activities are important for him/her to focus on during reablement is essential to achieving motivation and success in reablement. ${ }^{5,24}$ The relatives in our study also shared this view of goal-setting; however, it is a dilemma when the relatives think that the goals are unrealistic or too challenging to achieve. The adults undergoing reablement have their own perspectives of the situation; however, it is also important to listen to the relatives' perspectives without disempowering the older adult. The relatives wanted to be invited to share their perspectives on the older adult's goals. This is also confirmed in a study by Almborg et al, ${ }^{29}$ in which $\sim 80 \%$ of the relatives perceived no participation at all in determining the goals and needs of the discharge plans of stroke patients. However, Siegert and Taylor, ${ }^{30}$ argue that there can be discrepancies, and even conflict, between the goals that the patient, the family, and the rehabilitation team view as important or realistic. However, the different perspectives on goal setting have to be addressed through cooperation between the relatives, older adult, and professionals through open dialogue. ${ }^{30}$

Goal-setting is a "dynamic process that can be changed and adjusted according to progress." ${ }^{30}$ This requires the existence of processes and structures for decision making in which the voices of all parties are heard. According to international and national documents, ${ }^{3,7,8}$ collaborative practice will continue to become more embedded, strengthening health systems, and improving health outcomes. Thus, it is important and necessary for communities to ensure that there are opportunities for dialogue between relatives, older adults, and professionals. Additionally, these documents note that relatives are valued and highlighted as a cooperative component of the health care system.

\section{Wish to be a resource in reablement process - ethical dilemmas}

In our study, the relatives expressed several factors that are important to consider in collaborations. They want to be a resource and an important part of the process given the knowledge they have to share and to have the opportunity to participate in meetings. In the collaboration, professionals must use appropriate language with no professional arrogance. The relatives' capacity and resources to be in a partnership should be considered as well; otherwise, their participation might become a burden. However, all the relatives' preferences were clear that they want to collaborate and have an important role in their family members' reablement.

Several researchers ${ }^{11,12,17,31,32}$ argue that health care providers should focus on developing partnerships with family caregivers that recognize the active and essential role these individuals play in our health care system. This collaborative model recognizes and promotes the ability of caregivers to identify and articulate their needs and problems as they experience them. Although traditional rehabilitation models promote a collaborative approach, usually the patient is considered the active member, and the family caregiver is marginalized as a member of the team. Caregivers' needs for ongoing information, support, education, and skills training are overlooked. ${ }^{31,32}$ However, despite several studies that have noted how essential it is to establish a "real team" among all parties involved, the implementation of this concept in practice lags behind the ideal. ${ }^{32,33}$

The 3 months period of reablement in this study, is a longer prior of time than might be provided in ordinary reablement service in Norway. Such a period of professional and family caregiver contact should provide more opportunity for partnerships to work - however, in this case it did not. The relatives in our study called for a system, a routine, a culture, and an attitude for sharing information and knowledge. We argue that such a system and routine could move collaborative practices forward. As international political documents states, a framework for cooperation and decision-making 
between informal caregivers, older adults, and health care professionals is necessary. ${ }^{3,7}$ Our results also correspond to those of Brereton and Nolan ${ }^{32}$ and Cree et $\mathrm{al}^{12}$ who highlight that a collaborative practice implies mutual respect and requires confidence and trust in one's own and others' knowledge to develop a fruitful partnership. This is confirmed in studies by Wilde and Glendinning, ${ }^{2}$ Glendinning et al, ${ }^{19}$ and Rabbie and Glendinning, ${ }^{20}$ who argue that appreciating others' knowledge as a resource for the reablement process is essential. Lévesque et $\mathrm{al}^{11}$ advocate for the creation of an action plan following negotiation between the practitioner and the caregiver. The partnership process empowers the caregivers by allowing them to take an active role in the meeting and to realize that the professionals truly want to know their opinions. However, tensions between the older adults, the relatives, and the professionals could arise over the goals or the services proposed because the interests of each party do not always coincide. This ethical dilemma has to be resolved in a climate of trust and through the process of dialogue and negotiation between all parties involved in the decision. ${ }^{11}$ Partnership is an interactive process that fosters symmetry between the person in need of service, the relatives, and the professional team. However, whose point of view should be essential in situations in which dialogue and negotiations do not reach agreement? Professional judgment may be a solution, and that professional judgment must be justified based on each individual's situation and life history and on different perspectives. This is what Aristotele ${ }^{34}$ called "phronesis", that is, knowledge in terms of discretion and good judgment in action. ${ }^{35}$

Although official documents advocate for an even higher involvement of informal caregivers and promote "aging in place" for older people, ${ }^{3,7,8}$ van Wieringen et a ${ }^{10}$ noted that this policy means that the services formerly provided by formal care are now requested of informal caregivers or relatives. However, relatives cannot compensate for official caregiving, though their perspectives and voices must be reflected in the service provided. Furthermore, van Wieringen et a ${ }^{10}$ highlight that the renewed focus on caregiver involvement and the continuous budget cuts indicate that health care professionals and the health care system have to consider how to establish partnerships with relatives and enhance the quality of care. This partnership is expected to support both informal and formal care and to prevent informal caregivers from being overburdened. Our study revealed that the free time allows relatives to experience a break from providing care and eases the burden on relatives. The findings highlight the importance of taking care of this "room of freedom" because having some free time suggests that relatives have time to engage in their own meaningful activities and other activities necessary for everyday life. This is in line with relatives' experiences in a study by Wilde and Glendinning, ${ }^{2}$ who reported heavy caring responsibilities and the need for support and breaks in care.

\section{Follow-up programs}

Another interesting result in our study was that most of the participants wanted a follow-up program for their family members. They missed having a routine or a follow-up system to verify that the functions achieved by the older adults were maintained over time. According to Hjelle et al, ${ }^{24}$ some older adults continued engaging in physical exercise, while others did not because they no longer had someone to encourage and support them. The older adults had been collaborating with the multiprofessional team and the home-care personnel for up to 3 months and had thus built relationships. The abrupt ending meant they were feeling a loss. Further, the study of Hjelle et $\mathrm{a}^{24}$ revealed that health care personnel as well as relatives are important extrinsic motivators in reablement. Some older adults are in need of this extrinsic motivator for longer durations than others, and it is important that these persons are offered a follow-up program. This program has to be flexible and adjusted to the person's life situation. The results presented in our study provide knowledge that may assist in evaluating and developing reablement interventions tailored to the follow-up needs of older adults and their relatives.

In addition, this paper highlights the fact that relatives are an essential resource and collaborative component of the reablement team. These results provide and add knowledge that inform reablement teams as well as the health care system of the importance of collaborating with relatives. Despite the high satisfaction with the family members' progress in the reablement process, the relatives expressed unmet needs and concerns of participating in reablement that have to be considered in future developments of reablement services. Tensions between older adults, relatives and professionals could arise over the goals or the services proposed. This ethical dilemma needs to be resolved in a climate of trust, using dialogue and negotiation between all parties involved in the decision-making process.

\section{Methodological considerations}

With regard to the methods, the strength of our study was that the relatives of older adults who participated in reablement expressed their experiences and thus provided us with valuable insight. When researching and developing the notion of reablement, it is important to hear their voices and to express their experiences in their own words. 
Another strength of our study is that all the authors analyzed the transcribed data separately and together to ensure the trustworthiness of the analysis. Further, we have used representative text from the transcripts as quotations in the reporting of the results ${ }^{25,27}$ The quotes are not contextualized with specific data from the participants (i.e., men, woman, daughter, spouse, and age) due to the anonymity of the participants. They were recruited from a small community, and may have been recognized.

One limitation of the study is the fact that the empirical data are from a limited number of participants. This means that they do not necessarily represent other relatives of older adults participating in reablement service; rather, the study provided more in-depth insight into a few cases that could be related to similar situations or cases. ${ }^{21,25,26}$ For example, our findings of give and receive information, lack of collaboration, and conflicting expectations, may be recognizable for other relatives, as well as for multiprofessional team working in reablement service. According to Flick ${ }^{36}$ it must be noted that the generalization is not in every case the goal of a qualitative study.

A crucial question in qualitative research is how to decide when to stop integrating further cases. ${ }^{36}$ The criterion of "theoretical saturation", means that no additional data are being found and nothing new emerges any more. Though the sample size was not defined in advance, our preliminary and emerging data analyzing process revealed that none of the participants were invited to take part or collaborate with the team in the family member's reablement process. Several of the participants highlighted that they missed a system and a routine for collaboration. Since the reablement team obviously did not have this routine, we decided that saturation was achieved.

Another limitation of this study is that it provides a unilateral perspective. The reablement team was not interviewed on their perspectives of involving relatives in a collaborative reablement process. However, we decided that the relatives' opinions and experiences should be heard in this study.

\section{Conclusion}

The relatives experienced a lack of invitation from the reablement service to an open dialogue to give and receive information and knowledge about their family members. This paper adds to existing research on reablement, the importance of the relatives' wish to give and receive information, and being a resource in the reablement process to support and motivate their family members during the reablement process.

These results provide knowledge that can inform the reablement team as well as the health care system of the importance of collaborating with relatives. Despite the high satisfaction with their family members' progress in the reablement process, relatives expressed unmet needs, and concerns to be considered in future developments of reablement services. To clarify conflicting expectations from different parties, it was important for some relatives to make a conscious choice in terms of how to be involved in the reablement process. Ethical dilemmas such as conflict regarding the disempowerment and loss of autonomy of the older adult, have to be considered of the reablement team. The transition from reablement was too abrupt and the relatives advocated for the existence of an individualized follow-up program. Future research could explore the multidisciplinary teams' experiences of collaborating with the relatives and their perspectives of involving relatives in a collaborative reablement process. Additionally, research could further explore the relatives' experiences of collaborating with a reablement team, who have developed partnerships with family caregivers.

\section{Acknowledgments}

We thank all the participants who willingly shared their experiences of reablement with us. We also thank the project leaders of the reablement services for recruiting participants for this research.

\section{Authors' contributions}

$\mathrm{KMH}, \mathrm{HA}$, and OF participated in the design of the study. The interviews were conducted and transcribed verbatim by KMH. KMH, HA, and OF have made substantial contributions to analysis and interpretation of data. They have all been involved in drafting the manuscript, reading the manuscript, revising it critically, and have provided important comments to the content of all parts of the paper. All the authors have read and approved the final manuscript to be published.

\section{Disclosure}

$\mathrm{KMH}, \mathrm{HA}$, and the local research assistants were sponsored by Regional Research Funds Western Norway, grant number 229759. The authors report no conflicts of interest in this work.

\section{References}

1. Cochrane A, Furlong M, McGilloway S, Molloy DW, Stevenson M, Donnelly M. Time-limited home-care reablement services for maintaining and improving the functional independence of older adults. Cochrane Database Syst Rev. 2016;10:CD010825.

2. Wilde A, Glendinning C. If they're helping me then how can I be independent? The perceptions and experience of users of home-care re-ablement services. Health Soc Care Comm. 2012;20(6):583-590. 
3. UNECE. Innovative and empowering strategies for care. Policy Brief on Ageing No. 15. United Nations Economic Commission for Europe; 2015. Available from: https://www.unece.org/fileadmin/DAM/pau/age/ Policy_briefs/ECE-WG.1-21-PB15.pdf. Accessed December 12, 2016.

4. Ministry of Health and Care Services. Ansvar og meistring: mot ein heilskapleg rehabiliteringspolitikk: St.meld. $n r$ 21. [Responsibility and Coping: Towards a Holistic Rehabilitation Policy]. Oslo, Norway: Ministry; 1999. Norwegian.

5. Tuntland H, Aaslund MK, Espehaug B, Førland O, Kjeken I. Reablement in community-dwelling older adults: a randomised controlled trial. $B M C$ Geriatr. 2015;15(1):1-11.

6. UNECE. Dignity and non-discrimination for persons with dementia. Policy Brief on Ageing No. 16. United Nations Economic Commission for Europe; 2015. Available from: https://www.google.co.nz/ url? $\mathrm{sa}=\mathrm{t} \& \mathrm{rct}=\mathrm{j} \& \mathrm{q}=\& \mathrm{esrc}=\mathrm{s} \&$ source $=$ web $\& \mathrm{~cd}=1 \& \mathrm{cad}=\mathrm{rja} \& u a c t=8$ \&ved=0ahUKEwiT9au-te3QAhUEFJQKHdzgAh8QFggdMAA\&u $\mathrm{rl}=$ https $\% 3 \mathrm{~A} \% 2 \mathrm{~F} \% 2 \mathrm{Fwww}$. unece.org $\% 2$ Ffileadmin $\% 2 \mathrm{FDAM} \% 2$ Fpau\%2Fage\%2FPolicy_briefs\%2FECE-WG.1-23.pdf\&usg=AFQj CNGNHZPWKCux6ag55C3jfIQEPufXpQ\&sig2=1 YeikeaqVStjbOyT8p2Lg\&bvm=bv.141320020,d.dGc. Accessed December 12, 2016.

7. WHO. Framework for Action on Interprofessional Education \& Collaborative Practice. Geneve, Switzerland: WHO; 2010.

8. Ministry of health and care services. Fremtidens primarhelsetjenesterncerhet og helhet [The primary health and care services of tomorrow - localised and integrated]. Oslo, Norway: Ministry; 2014. Norwegian.

9. Helse-og Omsorgsdepartemente. Forskrift om habilitering og rehabilitering, individuell plan og koordinator [Regulations on habilitation and rehabilitation]. Ministry of Health: Norway; 2011. Norwegian.

10. van Wieringen M, Broese van Groenou MI, Groenewegen P. Impact of home care management on the involvement of informal caregivers by formal caregivers. Home Health Care Serv Q. 2015;34(2):67-84.

11. Lévesque L, Ducharme F, Caron C, Hanson E, Magnusson L, Nolan J, Nolan M. A partnership approach to service needs assessment with family caregivers of an aging relative living at home: a qualitative analysis of the experiences of caregivers and practitioners. Int J Nurs Stud. 2010; 47(7):876-887.

12. Cree L, Brooks HL, Berzins K, Fraser C, Lovell K, Bee P. Carers' experiences of involvement in care planning: a qualitative exploration of the facilitators and barriers to engagement with mental health services BMC Psychiatry. 2015;15(1):208.

13. Greenwood N, Mackenzie A. Informal caring for stroke survivors: meta-ethnographic review of qualitative literature. Maturitas. 2010; 66(3):268-276.

14. Gustafsson L, Bootle K. Client and carer experience of transition home from inpatient stroke rehabilitation. Disabil Rehabil. 2013;35(16):1380-1386.

15. Nolan M. Working with family carers: towards a partnership approach. Rev Clin Gerontol. 2001;11(01):91-97.

16. Greenwood N, Mackenzie A, Wilson N, Cloud G. Managing uncertainty in life after stroke: a qualitative study of the experiences of established and new informal carers in the first 3 months after discharge. Int J Nurs Stud. 2009;46(8):1122-1133.
17. Sadler E, McKevitt C. Expert carers: an emergent normative model of the caregiver. Soc Theory Health. 2013;11(1):40-58.

18. Larkin M, Milne A. Carers and empowerment in the UK: a critical reflection. Soc Policy Soc. 2014;13(01):25-38.

19. Glendinning C, Jones K, Baxter K, et al. Home Care Re-Ablement Services: Investigating the Longer-Term Impacts (prospective longitudinal $s t u d y$ ). New York, NY: University of York; 2010.

20. Rabiee P, Glendinning C. Organisation and delivery of home care re-ablement: what makes a difference? Health Soc Care Comm. 2011;19(5):495-503.

21. Sandelowski M, Leeman J. Writing usable qualitative health research findings. Qual Health Res. 2012;22(10):1404-1413.

22. Sandelowski M. Focus on research methods: whatever happened to qualitative description? Res Nurs Health. 2000;23(4):334-340.

23. Tuntland H, Espehaug B, Forland O, Hole AD, Kjerstad E, Kjeken I. Reablement in community-dwelling adults: study protocol for a randomised controlled trial. BMC Geriatr. 2014;14:139.

24. Hjelle KM, Tuntland H, Førland O, Alvsvåg H. Driving forces for home-based reablement; a qualitative study of older adults' experiences. Health Soc Care Community. Epub 2016 Jan 24.

25. Kvale S, Brinkmann S. Interviews: Learning the Craft of Qualitative Research Interviewing. 2nd ed. Los Angeles, CA: Sage; 2009.

26. Sandelowski M. What's in a name? Qualitative description revisited. Res Nurs Health. 2010;33(1):77-84.

27. Malterud K. Systematic text condensation: a strategy for qualitative analysis. Scand J Public Health. 2012;40(8):795-805.

28. Nolan M, Grant G, Keady J. Partnerships In Family Care: Understanding the Caregiving Career. Maidenhead: McGraw-Hill Education; 2003.

29. Almborg AH, Ulander K, Thulin A, Berg S. Discharge planning of stroke patients: The relatives' perceptions of participation. J Clin Nurs. 2009;18(6):857-865.

30. Siegert RJ, Taylor WJ. Theoretical aspects of goal-setting and motivation in rehabilitation. Disabil Rehabil. 2004;26(1):1-8.

31. Elliott TR, Pezent GD. Family caregivers of older persons in rehabilitation. NeuroRehabilitation. 2008;23(5):439-446.

32. Brereton L, Nolan M. Seeking partnerships between family and professional carers: stroke as a case in point. In: Nolan M, editor. Partnership in Family Care: Understanding the Caregiving Career. Maidenhead: McGraw-Hill Education; 2003:50-68.

33. Pickard S, Glendinning C. Comparing and contrasting the role of family carers and nurses in the domestic health care of frail older people. Health Soc Care Community. 2002;10(3):144-150.

34. Aristoteles. Nichomachean Ethics. Indianapolis, IN: Hackett Publisher; 1985.

35. Hjelle KM, Skutle O, Førland O, Alvsvåg H. The reablement team's voice: a qualitative study of how and integrated multidisciplinary team experiences participation in reablement. $J$ Multidiscip Healthc. 2016;9:575-585.

36. Flick U. An Introduction to Qualitative Research. Los Angeles, CA: Sage; 2009.
Journal of Multidisciplinary Healthcare

\section{Publish your work in this journal}

The Journal of Multidisciplinary Healthcare is an international, peerreviewed open-access journal that aims to represent and publish research in healthcare areas delivered by practitioners of different disciplines. This includes studies and reviews conducted by multidisciplinary teams as well as research which evaluates the results or conduct of such teams or health
Dovepress

care processes in general. The journal covers a very wide range of areas and welcomes submissions from practitioners at all levels, from all over the world The manuscript management system is completely online and includes a very quick and fair peer-review system. Visit http://www.dovepress.com/ testimonials.php to read real quotes from published authors. 